\title{
Immunohistochemical Discrimination Between the ASPL-TFE3 Fusion Proteins of Alveolar Soft Part Sarcoma
}

\author{
David T. Vistica, PhD,* Paula M. Krosky, PhD,† Susan Kenney, BS,* Mark Raffeld, MD, $\neq$ \\ and Robert H. Shoemaker, PhD*
}

\begin{abstract}
Summary: Alveolar soft part sarcoma (ASPS), a rare soft tissue sarcoma, is characterized by a chromosomal translocation $\operatorname{der}(17) t(\mathrm{X} ; 17)(\mathrm{p} 11 ; \mathrm{q} 25)$ resulting in the production of 2 fusion proteins encoded by regions of the genes for alveolar soft part locus (ASPL) and the transcription factor E3 (TFE3). In this study, polyclonal antibodies were generated to 25 mer peptides encompassing the junctional regions of ASPL-TFE3 type 1 and ASPL-TFE3 type 2 . The specificity of the affinity purified antibodies for the synthetic peptides and recombinant expressed ASPL-TFE3 type 1 and ASPL-TFE3 type 2 proteins was evaluated by enzyme-linked immunosorbent assay and was highly fusion type specific. Immunohistochemical staining of formalin-fixed, paraffin-embedded ASPS tumors with the fusion-specific antibodies resulted in intense nuclear staining and differentiation between tumors that express the type 1 protein and tumors that express the type 2 protein. These antibodies will be useful for the differential diagnosis of type 1 and type 2 ASPS and also in the detection of the fusion proteins in biochemical and cell biologic investigations.
\end{abstract}

Key Words: alveolar soft part sarcoma (ASPS), ASPL-TFE3 type 1 and 2 antibodies, gene fusion epitope detection

(J Pediatr Hematol Oncol 2008;30:46-52)

A lveolar soft part sarcoma (ASPS) is a rare neoplasm, constituting less than $1 \%$ of all soft tissue sarcomas. Originally described in 1952, ${ }^{1}$ this tumor is found

Received for publication July 10, 2007; accepted October 3, 2007.

From the *Screening Technologies Branch, Developmental Therapeutics Program, Division of Cancer Treatment and Diagnosis; †Applied/ Developmental Directorate, SAIC-Frederick Inc, National Cancer Institute at Frederick, Frederick; and \$Laboratory of Pathology, National Cancer Institute, National Institutes of Health, Bethesda, MD.

Funded in whole or in part with federal funds from the National Cancer Institute, National Institutes of Health, under contract N01-CO12400. The content of this publication does not necessarily reflect the views or policies of the Department of Health and Human Services, nor does the mention of trade names, commercial products, or organizations imply endorsement by the US Government. This research was supported by the Developmental Therapeutics Program in the Division of Cancer Treatment and Diagnosis of the National Cancer Institute.

Reprints: David T. Vistica, PhD, Screening Technologies Branch, Developmental Therapeutics Program, National Cancer Institute at Frederick, Bldg. 322/Room 104, Frederick, MD 21702 (e-mail: vistica@dtpax2.ncifcrf.gov).

Copyright $(\mathcal{2} 2008$ by Lippincott Williams \& Wilkins primarily in adolescents and young children. ASPS exhibits a highly characteristic histopathology, which is the basis for clinical diagnosis. Most notable are the alveolar architecture and the presence of cytoplasmic rhomboid crystals and granules that stain with periodic acid-Schiff (PAS) reagent and are resistant to digestion with diastase. ${ }^{2}$ ASPS characteristically exhibits a nonreciprocal chromosomal translocation, $\operatorname{der}(17) \mathrm{t}(\mathrm{X} ; 17)$ (p11;q25), ${ }^{3}$ and seminal work by Ladanyi and coworkers ${ }^{4}$ indicated that this translocation fuses the $\mathrm{N}$-terminal region of the alveolar soft part locus (ASPL) gene, located at $17 \mathrm{q} 25$, to the $\mathrm{C}$-terminal region of the transcription factor E3 (TFE3), located at Xp11. Two alternative fusion junctions have been observed resulting in the expression of 2 distinct fusion transcripts, ASPL-TFE3 type 1 and type 2 , and their respective proteins. The type 2 protein retains the primary TFE3 activation domain encoded by exon 5 , whereas this domain has been lost in the type 1 protein. One consequence of the ASPL-TFE3 gene fusion is increased nuclear reactivity to antibodies directed against TFE3, which suggests overexpression of the fusion protein relative to normal levels of TFE3. ${ }^{5}$ This result, however, provides no insight into potential differences in the pathobiologic effects of the 2 forms of the fusion protein. At the present time, it remains unclear whether different biologic and/or clinical consequences are associated with the 2 ASPL-TFE3 fusion proteins. Historically, generation of antibodies for immunocytochemical detection of junctional epitopes of chimeric proteins produced as a result of chromosomal translocations has proved to be exceedingly difficult. ${ }^{6}$ Nonetheless, in an effort to develop reagents that allow detection and differentiation between ASPL-TFE3 type 1 and ASPLTFE3 type 2 fusion proteins, we sought to develop antibodies against 25 mer peptides homologous to the ASPL-TFE3 fusion domains. Presently, we report that the resulting antibodies are able to discriminate between synthetic ASPL-TFE3 type 1 and type 2 fusion peptides, recombinant ASPL-TFE3 fusion proteins and the 2 fusion proteins in formalin-fixed, paraffin-embedded ASPS tumor tissue.

\section{MATERIALS AND METHODS}

\section{ASPS Tumor Acquisition and RNA Preparation}

ASPS tumors were obtained from surgery following informed consent under NCI clinical research protocol $05-\mathrm{C}-\mathrm{N} 138$. A representative tumor sample $(0.5$ to $1.0 \mathrm{~cm})$ 
was fixed in buffered $10 \%$ formalin for confirmatory histopathology including hematoxylin and eosin, PAS/ diastase, and immunohistochemical staining for TFE3 (sc-5958, Santa Cruz Biotechnology, Santa Cruz, CA). A portion of tumor tissue was immediately placed in RNAlater solution (Ambion, Austin, TX), stored at $4{ }^{\circ} \mathrm{C}$ and subsequently total RNA was isolated using the RNeasy Fibrous Midi Kit (Qiagen, Valencia, CA) according to the manufacturer's instructions. The concentration of RNA was determined on a Nanodrop spectrophotometer, and the A260/A280 values were greater than 1.9. The integrity and size distribution of RNA were evaluated on $1 \%$ denaturing NuSieve agarose gels (Cambridge, Rockland, ME) followed by ethidium bromide staining.

\section{Reverse Transcription/Polymerase Chain Reaction Detection of ASPL-TFE3 Type 1 and Type 2 Fusion Transcripts}

Reverse transcription was performed using the Superscript First Strand Synthesis system (Invitrogen, Carlsbad, CA) according to the manufacturer's instructions. Briefly, total RNA $(1 \mu \mathrm{g})$ was denatured and annealed to random hexamers before the addition of RT buffer, $\mathrm{MgCl}_{2}$, dithiothreitol, an RNAse inhibitor, and reverse transcriptase. Elongation was performed at $42^{\circ} \mathrm{C}$ for 30 minutes, followed by inactivation of the reverse transcriptase at $99^{\circ} \mathrm{C}$ for 5 minutes. A negative control of diethylpyro carbonate-treated water and a positive control of total HeLa RNA were included in each experiment. The resulting cDNAs were diluted with a polymerase chain reaction (PCR) mastermix that included PCR buffer, $\mathrm{MgCl}_{2}$, dNTPs, primers $(1.5 \mu \mathrm{M}$ each), and Taq DNA polymerase. PCR consisted of 40 cycles of $95^{\circ} \mathrm{C} / 60$ seconds, $60^{\circ} \mathrm{C} / 60$ seconds, and $72^{\circ} \mathrm{C} / 60$ seconds, followed by a final extension at $72^{\circ} \mathrm{C}$ for 10 minutes. The PCR products were electrophoresed in $2 \%$ NuSieve agarose gel (Cambrex, Rockland, ME) and stained with ethidium bromide. ASPL-TFE3 fusion transcripts were detected using a forward primer corresponding to nt 548 to 569 of ASPL (AAAG AAGT CCAA GTCGG GCCA) and a reverse primer corresponding to nt 972 to 993 in exon 4 of TFE3 (CGTT TGAT GTTG GGCA GCTCA) ${ }^{4}$ (Integrated DNA Technologies Inc, Coralville, IA). Plasmids encoding ASPL-TFE3 type 1 and ASPL-TFE3 type 2 were kindly provided by Dr Marc Ladanyi, Memorial Sloan Kettering Cancer Center and were used as positive controls. Expected fragment sizes were $195 \mathrm{bp}$ for ASPL-TFE3 type 1 and $300 \mathrm{bp}$ for ASPL-TFE3 type 2.

\section{Antibody Preparation}

Polyclonal antibodies were raised in rabbits against a 25 mer fusion peptide comprised of amino acids 302 to 311 (DPQQEQERER) of ASPL protein and either amino acids 296 to 310 (LPVSGNLLDVYSSQG) (ASPL-TFE3, type 1) or amino acids 261 to 275 (IDDVIDEIISLESSY) (ASPL-TFE3, type 2) of TFE3 (Invitrogen). Antibodies were affinity purified and used at a dilution of 1:5000 to
10,000 for tissue staining. Antibody to TFE3, prepared in goats, was obtained from Santa Cruz Biotechnology (sc5958) and was also used at a dilution of 1:5000 to 10,000.

\section{Immunohistochemical Staining}

Tissue sections, $4-\mu \mathrm{m}$ affixed to glass slides, were deparaffinized and treated for 15 minutes in methanol containing $0.6 \% \mathrm{H}_{2} \mathrm{O}_{2}$ followed by $1 \mathrm{mM}$ ethylene diamine tetraacetic acid ( $\mathrm{pH} 8.0)$ in a microwave vacuum histoprocessor (RHS-1; Milestone, Italy). The temperature was gradually increased to $100^{\circ} \mathrm{C}$ over 10 minutes and maintained at $100^{\circ} \mathrm{C}$ for an additional 10 minutes. Sections were cooled, rinsed sequentially in $\mathrm{H}_{2} \mathrm{O}$ and phosphate-buffered saline (PBS) for 10 minutes each, and blocked with $10 \%$ normal rabbit serum (TFE3 antibody) or 2\% serum (ASPL-TFE3 type 1 and 2 antibodies) in PBS for 20 minutes. Primary antibody, used at a dilution of 1:5000 to 10,000 in PBS containing $0.1 \%$ bovine serum albumin (BSA), was overlayed onto the tissue sections overnight at $4^{\circ} \mathrm{C}$. Sections were rinsed in PBS for 10 minutes and incubated with biotinylated secondary antibody (antigoat for TFE3 and antirabbit for ASPL-TFE3 type 1 and ASPL-TFE3 type 2 fusion proteins) in PBS containing $1.5 \%$ normal serum for 30 minutes. Sections were rinsed in PBS for 10 minutes and processed using the Vectastain Elite ABC kit (Vector Laboratories, Burlingame, CA) according to the manufacturer's instructions.

\section{Cloning, Expression, and Purification of Recombinant Proteins}

TFE3, ASPL-TFE3 type 1, and ASPL-TFE3 type 2 genes were subcloned by PCR from plasmids kindly provided by Dr Marc Ladanyi, Memorial Sloan Kettering Cancer Center. Fragments were first amplified for 5 cycles using $200 \mathrm{nM}$ each of primers L1339 and L1341 (for TFE3) or L1340 and L1341 (for ASPL-TFE3 fusions) (for sequences, see below). After this, $200 \mathrm{nM}$ of adapter primer L907 was added, and amplification was continued for 15 cycles. PCR was carried out using Platinum Taq HiFidelity (Invitrogen) under standard conditions using a 2-minute extension time. The final PCR products contain the genes of interest flanked on the $5^{\prime}$ side with a Gateway attB1 site and Tobacco Etch Virus protease cleavage site. The $3^{\prime}$ side contains a Gateway attB2 site. The PCR products were purified using the QiaQuick PCR purification kit (Qiagen) and recombined into pDonr223 using the Gateway BP recombination reaction (Invitrogen) and the manufacturer's protocols. pDonr223 is a modified version of pDonr201 (Invitrogen) in which the kanamycin resistance gene was replaced with a gene encoding spectinomycin resistance and several additional sequencing primer sites were added to aid in sequence verification of entry clones. The resulting entry clones were completely sequence verified, and selected entry vectors were transferred to pDest544 using Gateway LR Clonase (Invitrogen). Final expression clones encode proteins of the form His6-NusA-B1-tev-TFE-stop. The expression plasmids were transfected into Escherichia coli Rosetta (DE3) (Novagen, Madison, WI), and overnight cultures 
were diluted 1:20 in Super Broth (Quality Biological Inc, Gaithersburg, MD) with $100 \mu \mathrm{g} / \mathrm{mL}$ of ampicillin (Sigma), grown to $\mathrm{OD} \sim 0.5$, and induced with $0.5 \mathrm{mM}$ of isopropylthioglactoside (Sigma) for 4 hours at $20^{\circ} \mathrm{C}$. Cells pellets were resuspended in BugBuster (Novagen) with complete protease inhibitors (Roche Applied Science, Indianapolis, IN) and stored at $-80^{\circ} \mathrm{C}$. Thawed lysates were sonicated to reduce viscosity and brought to $500 \mathrm{mM}$ of $\mathrm{NaCl}$ and $45 \mathrm{mM}$ of imidazole. The lysates were clarified at $50,000 \times \mathrm{g}$ for 20 minutes and loaded onto HisTrap FF crude columns (GE Biosciences, Piscataway, NJ). The columns were washed with 5 volumes of $50 \mathrm{mM}$ Tris, $\mathrm{pH} 8.0,500 \mathrm{mM}$ of $\mathrm{NaCl}$, and $60 \mathrm{mM}$ of imidazole, and protein was eluted with a gradient of the same buffer to $500 \mathrm{mM}$ imidazole. Selected fractions were pooled, diluted to $300 \mathrm{mM}$ of $\mathrm{NaCl}$ with $50 \mathrm{mM}$ Tris, $\mathrm{pH} 8.0$ with $0.01 \%$ Brij-35 (Buffer A), bound to a HiTrap heparin HP column (GE Biosciences), and eluted with a gradient to $1 \mathrm{M}$ of $\mathrm{NaCl}$ in Buffer A. Selected fractions were pooled, diluted to $250 \mathrm{mM}$ of $\mathrm{NaCl}$ with Buffer A, mixed with Tobacco Etch Virus (Invitrogen, Carlsbad, CA) and $0.5 \mathrm{mM}$ of dithiothreitol, and cleaved overnight at room temperature. The linker between the fusion tag and the gene of interest consists of the amino acid sequence ENLYFQG, which is cleaved by Tev protease between the $\mathrm{Q}$ and $\mathrm{G}$ residues to leave only a single glycine at the amino terminus of the protein of interest.

Residual fusion protein, NusA tag, and protease were removed with a second His-Trap column. Purified protein, located in the flow through, was quantified with the BioRad protein assay, aliquoted, and stored at $-80^{\circ}$.

The following oligonucleotides (Operon Inc) were used in this study:

L1339: 5'GGCGAAAACCTGTACTTCCAAGGCA

TGTCTCATGCGGCCGAACCAGCTCGGG

L1340: 5'GGCGAAAACCTGTACTTCCAAGGCA

TGGCGGCCCCGGCAGGCGGCGGAGGCT

L1341: 5'-GGGGACAACTTTGTACAAGAAAGTT

GGCTAGGACTCCTCTTCC

L907: 5'-GGGGACAACTTTGTACAAAAAAGTT

GGCGAAAACCTGTACTTCCAAGGC

\section{Quantification of Antibody Affinity}

Selected antigens $(100 \mu \mathrm{L}$ containing $0.05,0.1,0.25$, 0.50 , or $1.0 \mathrm{pMol}$ ) were added to 96-well EIA/RIA flat bottom Elisa plates (Corning/Costar, Corning, NY) and incubated at $4^{\circ} \mathrm{C}$ overnight in a humidified atmosphere. Six replicate wells were used for each concentration of peptide, protein, and antibody. Plates were washed 5 times for 1 minute each with $200 \mu \mathrm{L} /$ well PBS containing $0.05 \%$ Tween-20, blocked by addition of $200 \mu \mathrm{L} /$ well $1 \%$ BSA in PBS at $37^{\circ} \mathrm{C}$ for 60 minutes and then washed as before. Primary antibody was diluted in PBS with $1 \%$ BSA $(1: 100,1: 500,1: 1000,1: 5000$, and 1:10,000), the dilutions were added to the plates $(100 \mu \mathrm{L} /$ well $)$, and the plates were incubated at $37^{\circ} \mathrm{C}$ for 60 minutes and washed. Goat antirabbit IgG conjugated with Horseradish
Peroxidase (Santa Cruz, Santa Cruz, CA) was diluted 1:1000 in PBS containing 1\% BSA, an aliquot was added to each well $(100 \mu \mathrm{L})$ and the plates were incubated for 60 minutes at $37^{\circ} \mathrm{C}$ and washed. Peroxidase substrate was prepared in $0.1 \mathrm{M}$ of $\mathrm{Na}_{2} \mathrm{HPO}_{4} / 0.1 \mathrm{M}$ of sodium citrate $(\mathrm{pH}$ ) by addition of $4 \mathrm{mg} \mathrm{O}$ phenylenediamine to $10 \mathrm{~mL}$ buffer followed by $3.3 \mu \mathrm{L} 30 \% \mathrm{H}_{2} \mathrm{O}_{2}$. The substrate solution was added to wells $(100 \mu \mathrm{L} /$ well $)$ and $\mathrm{A} 405 \mathrm{~nm}$ read at intervals of 2.5 minutes for 60 minutes on a Bio Tek EL $\times 800$ plate reader (Bio-Tek Instruments Inc, Winooski, VT).

\section{Nonlinear Analysis of Binding Data}

Enzyme-linked immunosorbent assay (ELISA) data from each of the 6 replicate wells were fitted to an exponential nonlinear model using Statistical Analysis Software Nonlinear:

$$
\text { Optical density }(\text { od })=B+A\left(1-e^{-\alpha t}\right) \text {. }
$$

The derivative with respect to time was evaluated at $t=0(\mathrm{~A} \times \alpha)$ and provided an estimate of initial velocity of the reaction. These estimates were modeled via the The Michaelis-Menton (M-M) equation as a function of peptide or protein concentration:

$$
\text { Initial velocity }=
$$

Vmax [concentration/(Km + concentration)].

The 2 parameters were estimated using Statistical Analysis Software Nonlinear and a separate fit for each antibody concentration was carried out to check for the consistency of the estimates of the parameter $\mathrm{Km}$. An overall estimate of $\mathrm{Km}$ was made by pooling the data over antibody concentrations.

\section{RESULTS}

\section{Antibody Specificity for ASPL-TFE3 Type 1 and ASPL-TFE3 Type 2 Fusion Peptides and Recombinant Proteins}

The nonreciprocal chromosomal translocation $\operatorname{der}(17) t(X ; 17)(p 11 ; q 25)$, which is characteristic of ASPS, results in the expression of 1 of 2 fusion proteins that include the N-terminal 311 amino acids of ASPL and variable regions from the C-terminus of the TFE3. ASPLTFE3 type 1 retains aa 295 to 575 of TFE3 and the slightly longer ASPL-TFE3 type 2 retains aa 260 to 575 from TFE3. In an attempt to immunologically differentiate between these 2 fusion proteins, we synthesized 25 mer peptides encompassing the ASPL-TFE3 fusion junctions (Fig. 1). The 2 peptides shared an amino terminal 10 residues (aa 302 to 311 from ASPL) and differed in the residues derived from TFE3 (aa 296 to 310 for the type 1 peptide and aa 261 to 275 for the type 2 peptide). Two rabbits were immunized with each peptide, and the collected sera were affinity purified using the respective peptides. However, it was not clear if these antibodies would recognize their respective epitopes within the context of folded, full length proteins or if they could differentiate between the 2 variants of ASPL-TFE3, 


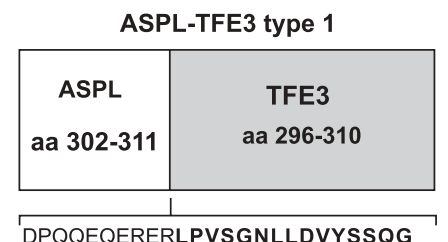

DPQQEQERERLPVSGNLLDVYSSQG

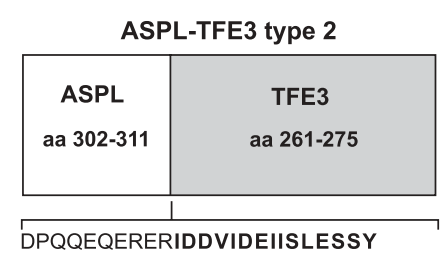

FIGURE 1. Amino acid sequences of ASPL-TFE3 type 1 and ASPL-TFE3 type 2 fusion peptides. ASPL-TFE3 type 1 peptide is comprised of aa 302 to 311 of ASPL and aa 296 to 310 of TFE3. ASPL-TFE3 type 2 peptide consists of aa 302 to 311 of ASPL and aa 261 to 275 of TFE3.

which have identical ASPL-derived N-terminal sequences. An ELISA was developed and used to measure the relative affinity of each antibody for the synthetic peptides. Each of the 2 antibodies was tested against the 2 synthetic peptides, and each antibody bound exclusively to its "matched peptide" (Table 1). No cross-reactivity was observed. Nonlinear analysis of the data generated a $K \mathrm{~m}$ of $11.3 \mathrm{nM}$ for the type 1 interaction and a $\mathrm{Km}$ of $1.6 \mathrm{nM}$ for the type 2 interaction. Thus, both antibodies were highly specific, and the ASPL-TFE 3 type 2 antibody exhibited a 7-fold greater affinity for its respective matched peptide than did the type 1 antibody.

The ELISA was also used to examine antibody binding, specificity, and affinity to recombinantly expressed ASPL-TFE3 type 1 and ASPL-TFE3 type 2 proteins. The ASPL-TFE3 type 2 antibodies recognized only the ASPL-TFE3 type 2 protein with a $K \mathrm{~m}$ of $1.35 \mathrm{nM}$, a value similar to the $K \mathrm{~m}$ observed for the interaction between this antibody and the type 2 peptide. The ASPL-TFE3 type 1 antibody recognized both ASPLTFE3 type 1 and 2 proteins but had substantially greater affinity (12.5-fold) for the ASPL-TFE3 type 1 recombi-

TABLE 1. Affinity of ASPL-TFE3 Type 1 and ASPL-TFE3 Type 2 Antibodies for ASPL-TFE3 Fusion Peptides and Proteins

\begin{tabular}{lccc}
\hline & Antibody & $\mathbf{K m}, \mathbf{n M}(\mathbf{S E})$ & $\mathbf{1 / K \mathbf { m }}$ \\
\hline ASPL-TFE3 fusion peptide & & & \\
Type 1 & Type 1 & $11.30(1.9)$ & 0.09 \\
Type 1 & Type 2 & $*$ & $*$ \\
Type 2 & Type 2 & $1.60(0.34)$ & 0.63 \\
Type 2 & Type 1 & $*$ & $*$ \\
ASPL-TFE3 recombinant protein & & & \\
Type 1 & Type 1 & $0.50(0.12)$ & 2.0 \\
Type 1 & Type 2 & $*$ & $*$ \\
Type 2 & Type 2 & $1.35(0.24)$ & 0.73 \\
Type 2 & Type 1 & $6.30(2.5)$ & 0.16 \\
\hline
\end{tabular}

$K \mathrm{~m}$ values were derived from ELISA quantification as described in Methods.

*Indicates no observable reaction.

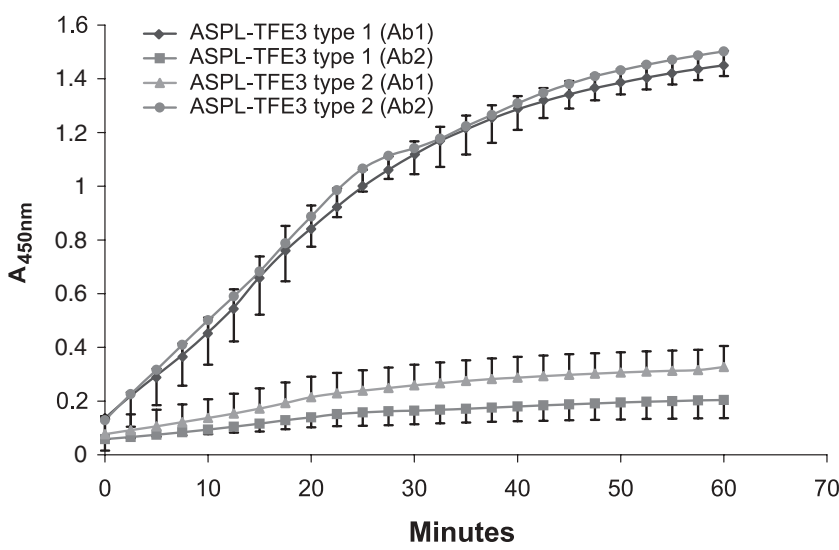

FIGURE 2. Specificity of ASPL-TFE3 type 1 and ASPL-TFE3 type 2 antibodies for ASPL-TFE3 type 1 and ASPL-TFE3 type 2 recombinant proteins. Selected concentrations of antigen were bound to EIA/RIA plates, exposed to listed antibodies, and developed with colorimetric reagents. Absorbance was read every 2.5 minutes for 60 minutes and replicate wells $(n=6)$ were averaged.

nant protein. Figure 2 illustrates the comparative specificity of both antibodies for ASPL-TFE3 type 1 and ASPL-TFE3 type 2 recombinant proteins.

\section{Immunohistochemical Staining of ASPL-TFE3 Type 1 and ASPL-TFE3 Type 2 ASPS Tumors}

The specificity of the ASPL-TFE3 type 1 and 2 antibodies was examined in representative formalin-fixed, paraffin-embedded ASPS tumors. Both type 1 and type 2 ASPS tumors exhibited characteristic histology consisting of organoid nests of cells, PAS positive/diastase resistant stained regions (Figs. 3A, 4A), and intense nuclear staining with an antibody raised against a C-terminal portion of TFE3, which is present in both type 1 and type 2 tumors (Figs. 3C, 4C). Tumors were classified as ASPLTFE3 type 1 (Fig. 3) or ASPL-TFE3 type 2 (Fig. 4) by reverse transcription-PCR (RT-PCR) detection of ASPLTFE3 type 1 or type 2 fusion transcript (panel B). Twelve cases were studied by immunohistochemistry and included 4 ASPL-TFE3 type 1 and 8 ASPL-TFE3 type 2 tumors as determined by RT-PCR. In addition, tumor samples from 2 of the type 1 and 2 of the type 2 cases were obtained from subsequent resection of tumor thus yielding 16 total tumor samples for analysis. Sections from these tumors were stained with the antibodies to the ASPL-TFE3 type 1 and type 2 peptides and differentiation was readily observed between the tumor and stromal cells and between type 1 and type 2 ASPS tumors (panels D, E). All 4 of the type 1 cases and all 8 of the type 2 cases stain specifically with their respective antibody. The ASPL-TFE3 type 2 antibody was highly specific for the ASPL-TFE3 type 2 cases, and showed no cross-reaction with the ASPL-TFE3 type 1 cases. Although the ASPLTFE3 type 1 antibody strongly stained the nuclei of ASPL-TFE3 type 1 cases, it also showed weak nuclear staining in of ASPL-TFE3 type 2 cases, paralleling the cross-reaction seen in the ELISA study. Nonetheless 


\section{ASPL-TFE3 type 1}
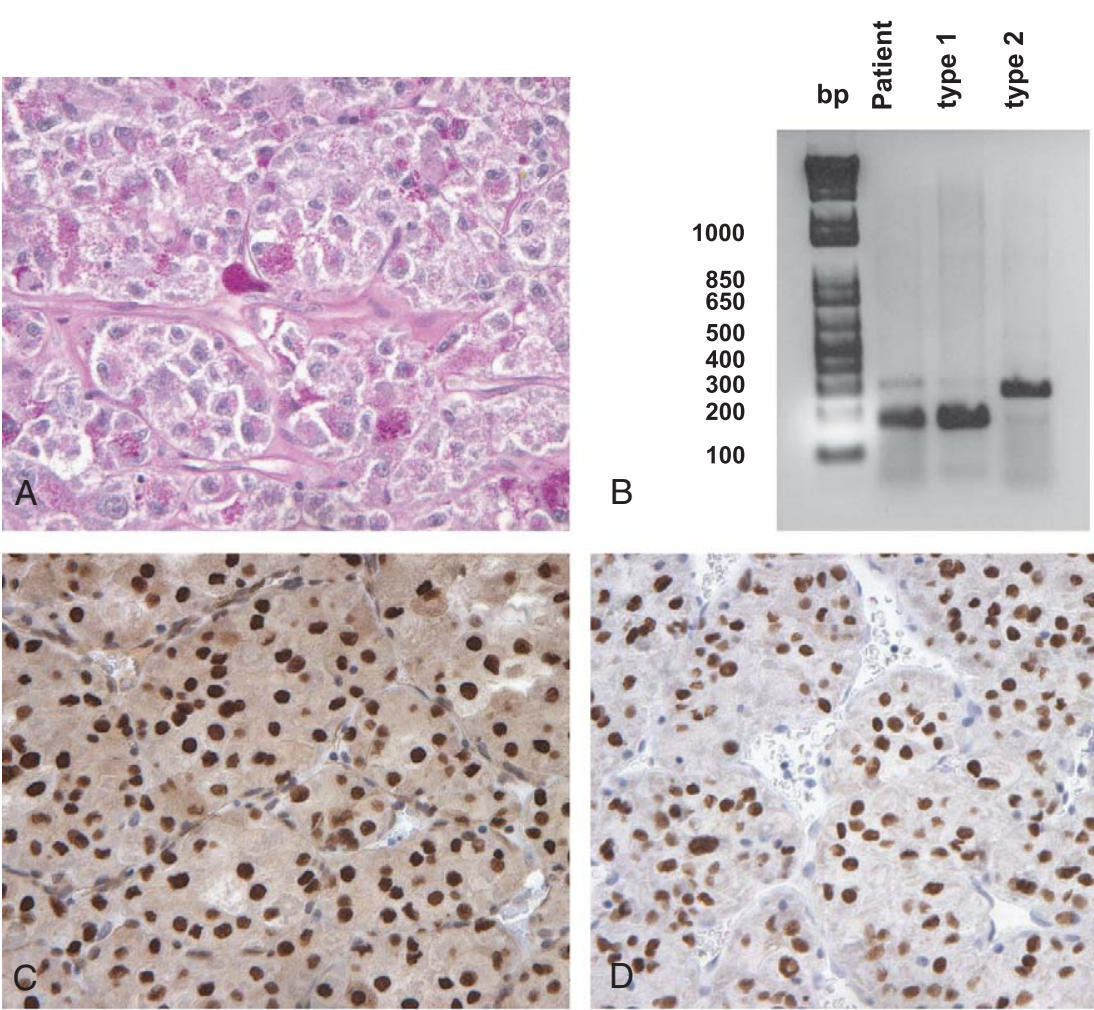

B

FIGURE 3. Histology, molecular classification, and immunohistochemistry of type 1 ASPS. All antibodies were used at a concentration of $1: 10,000$. A, PAS/ diastase. B, ASPL-TFE3 fusion transcript. C, TFE3 antibody. D, ASPL-TFE3 type 1 antibody. E, ASPL-TFE3 type 2 antibody. F, Negative control for ASPL-TFE3 type 1 antibody.
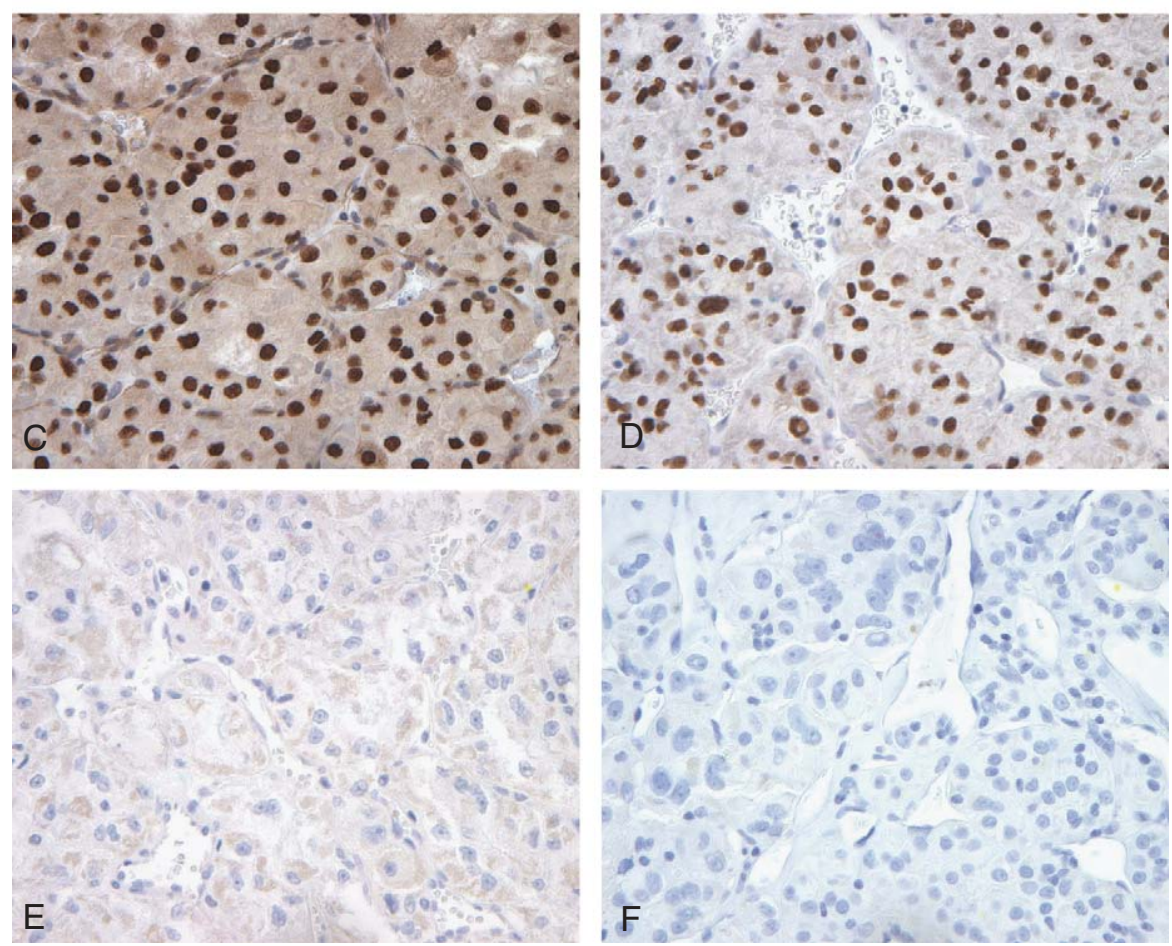

combined use of these 2 antibodies allowed easy identification of type 1 and type 2 cases.

\section{DISCUSSION}

The present investigation was undertaken to develop antibodies that allow detection of and discrimination between the 2 ASPL-TFE3 fusion proteins, resulting from the $\operatorname{der}(17) \mathrm{t}(\mathrm{X} ; 17)(\mathrm{p} 11 ; \mathrm{q} 25)$ chromosomal translocations associated with ASPS $^{3}$ and this was achieved. ELISA analysis of binding to synthetic peptides and recombinant proteins and immunohistochemical staining of formalinfixed, paraffin-embedded ASPS tumor tissue support this conclusion. Reports of antibodies against junctional epitopes of proteins produced as a result of chromosomal translocations have been rare. ${ }^{6}$ The difficulty of producing antibodies that recognize junctional epitopes within chimeric proteins can be attributed to several issues, including a potential lack of immunogenicity of the peptide sequence and potential differences in tertiary structure between immunogen peptides and full length proteins. These limitations can result in either a lack of recognition of the junctional epitope or a reduced affinity for the epitope. The first successful antibody to a fusion protein junction to be described targeted the E2A/pbx 1 fusion protein of the $\mathrm{t}(1 ; 19)(\mathrm{q} 23 ; \mathrm{p} 13.3)$ translocation found in acute lymphoblastic leukemia. ${ }^{7}$ A recent publication $^{8}$ reported the generation of monoclonal antibodies against 2 fusion proteins, TLS-CHOP and EWS-CHOP, which result from chromosomal translocations associated with myxoid and round cell liposarcomas, respectively. Emerging data indicate that the 2 ASPL-TFE3 fusion proteins function as aberrant transcription factors activating MET signaling ${ }^{9}$ and likely 
ASPL-TFE3 type 2
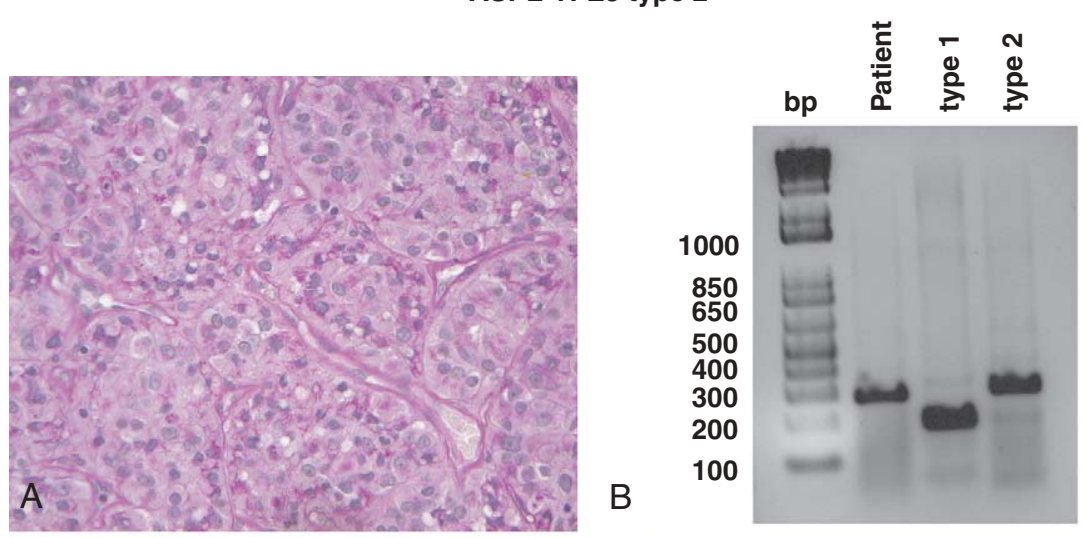

FIGURE 4. Histology, molecular classification, and immunohistochemistry of type 2 ASPS. A, PAS/diastase. B, ASPLTFE3 fusion transcript. C, TFE3 antibody. D, ASPL-TFE3 type 1 antibody. E, ASPLTFE3 type 2 antibody. F, Negative control for ASPL-TFE3 type 2 antibody.
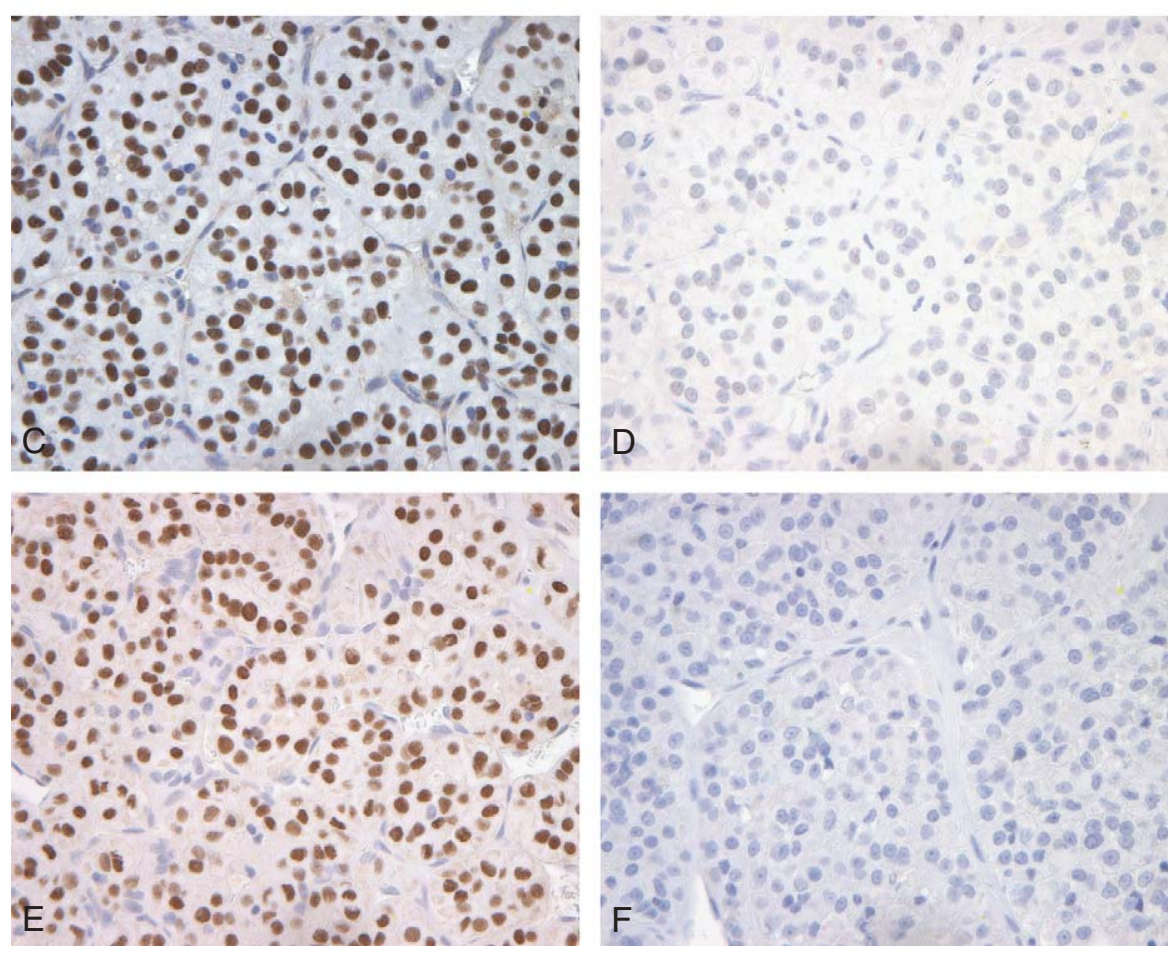

other important signal transduction pathways. It remains unclear whether different biologic consequences are associated with the 2 ASPL-TFE3 fusions. In contrast to the as yet undefined role of the 2 ASPL-TFE3 fusion proteins in ASPS, the PRCC-TFE3 fusion proteins associated with papillary renal carcinoma have been shown to have transforming potential. ${ }^{10}$ Recently Mathur and Samuels ${ }^{11}$ showed that the PSF-TFE3 fusion could transform $3 \mathrm{~T} 3$ cells and act as a functional transcription factor. Indeed fusions of TFE3 with yet additional genes are associated with renal cancers ${ }^{12-14}$ and supports the notion that aberrant transcription mediated by the TFE3 constituent of the fusion protein plays a key role in the pathogenesis of these tumors.

The antibodies described here, which recognize the ASPL-TFE3 type 1 and ASPL-TFE3 type 2 fusions, allow immunologic differentiation between and therefore facile diagnosis of type 1 and type 2 ASPS. The observation that the entire tumor cell population seems to express a fusion protein provides further evidence for a key role in the pathogenesis of ASPS. It remains to be determined whether the differences in the 2 fusion proteins confer different biologic consequences. Because the ASPL-TFE3 type 2 fusion protein contains a larger contribution from TFE3, including the activation domain, the biologic consequences of binding to its cognate DNA sequence may be different than that of the ASPLTFE3 type 1 fusion protein. Elimination of this domain from the native TFE3 gene confers a dominant negative phenotype. ${ }^{15}$ Such a radical phenotype seems not to be associated with the ASPL-TFE3 type 1 fusion protein. The presence or absence of sites of posttranslational modifications could also result in functional differences between the proteins. Sumoylation has been shown to be 
an important feature of TFE3 and the related transcription factors MITF and TFEB with which it can heterodimerize. ${ }^{16}$ Recent studies have provided additional information regarding the molecular mechanisms by which TFE3 can affect control of cellular growth. Nijman et $\mathrm{al}^{17}$ conducted a functional genetic screen, which revealed that TFE3 regulates cyclin $\mathrm{E}$ expression in an E2F dependent manner. The importance of adjacent E2F sites in promoters for transcriptional activation by the various TFE3 fusions remains to be established. Likewise, the relative ability of the fusion proteins to bind TFE3 partners such as lymphoid enhancer binding factor $1,{ }^{18}$ is unknown and could contribute to differences in the biologic behavior of the respective tumors. Given the very small number of ASPS tumors that have been characterized for fusion type to date and their heterogeneous presentation, it is not surprising that prognostic significance has not yet been recognized. Study of additional cases using molecular and immunohistochemical methods may support this in the future and contribute to the development of additional insight into the molecular interactions of these fusion proteins and suggest avenues for therapeutic intervention.

\section{ACKNOWLEDGMENTS}

The authors thank Ms Donna Butcher for the immunohistochemical staining, Dr Dominic Esposito for preparation of the protein expression constructs, Dr David Hoberman and Matthew Fivash for analysis of the antibody ELISA data, and Dr Luke Stockwin for critical evaluation of the manuscript.

\section{REFERENCES}

1. Christopherson WM, Foote FW Jr, Stewart FW. Alveolar soft-part sarcomas; structurally characteristic tumors of uncertain histogenesis. Cancer. 1952;5:100-111.

2. Shipkey FH, Lieberman PH, Foote FW Jr, et al. Ultrastructure of alveolar soft part sarcoma. Cancer. 1964;17:821-830.

3. Heimann P, Devalck C, Debusscher C, et al. Alveolar soft-part sarcoma: further evidence by FISH for the involvement of chromosome band 17q25. Genes Chromosomes Cancer. 1998;23:194-197.

4. Ladanyi M, Lui MY, Antonescu CR, et al. The der(17)t(X;17)(p11;q25) of human alveolar soft part sarcoma fuses the TFE3 transcription factor gene to ASPL, a novel gene at 17q25. Oncogene. 2001;20: 48-57.

5. Argani P, Lal P, Hutchinson B, et al. Aberrant nuclear immunoreactivity for TFE3 in neoplasms with TFE3 gene fusions: a sensitive and specific immunohistochemical assay. Am J Surg Pathol. 2003;27: 750-761.

6. Falini B, Mason DY. Proteins encoded by genes involved in chromosomal alterations in lymphoma and leukemia: clinical value of their detection by immunocytochemistry. Blood. 2002;99:409-426.

7. Sang BC, Shi L, Dias P, et al. Monoclonal antibodies specific to the acute lymphoblastic leukemia $\mathrm{t}(1 ; 19)$-associated E2A/pbx1 chimeric protein: characterization and diagnostic utility. Blood. 1997;89: 2909-2914.

8. Oikawa K, Ishida T, Imamura T, et al. Generation of the novel monoclonal antibody against TLS/EWS-CHOP chimeric oncoproteins that is applicable to one of the most sensitive assays for myxoid and round cell liposarcomas. Am J Surg Pathol. 2006;30: 351-356.

9. Tsuda M, Davis IJ, Argani P, et al. TFE3 fusions activate MET signaling by transcriptional up-regulation, defining another class of tumors as candidates for therapeutic MET inhibition. Cancer Res. 2007;67:919-929.

10. Weterman MA, van Groningen JJ, den Hartog A, et al. Transformation capacities of the papillary renal cell carcinomaassociated PRCCTFE3 and TFE3PRCC fusion genes. Oncogene. 2001;20:1414-1424.

11. Mathur M, Samuels HH. Role of PSF-TFE3 oncoprotein in the development of papillary renal cell carcinomas. Oncogene. 2007;26: 277-283.

12. Skalsky YM, Ajuh PM, Parker C, et al. PRCC, the commonest TFE3 fusion partner in papillary renal carcinoma is associated with pre-mRNA splicing factors. Oncogene. 2001;20:178-187.

13. Heimann P, El Housni H, Ogur G, et al. Fusion of a novel gene, $\mathrm{RCC} 17$, to the TFE3 gene in $\mathrm{t}(\mathrm{X} ; 17)(\mathrm{p} 11.2 ; \mathrm{q} 25.3)$-bearing papillary renal cell carcinomas. Cancer Res. 2001;61:4130-4135.

14. Argani P, Lui MY, Couturier J, et al. A novel CLTC-TFE3 gene fusion in pediatric renal adenocarcinoma with $\mathrm{t}(\mathrm{X} ; 17)(\mathrm{p} 11.2 ; \mathrm{q} 23)$. Oncogene. 2003;22:5374-5378.

15. Roman C, Cohn L, Calame K. A dominant negative form of transcription activator mTFE3 created by differential splicing. Science. 1991;254:94-97.

16. Miller AJ, Levy C, Davis IJ, et al. Sumoylation of MITF and its related family members TFE3 and TFEB. $J$ Biol Chem. 2005;280: $146-155$.

17. Nijman SM, Hijmans EM, El Messaoudi S, et al. A functional genetic screen identifies TFE3 as a gene that confers resistance to the anti-proliferative effects of the retinoblastoma protein and transforming growth factor-beta. J Biol Chem. 2006;281:21582-21587.

18. Yasumoto K, Takeda K, Saito H, et al. Microphthalmia-associated transcription factor interacts with LEF-1, a mediator of Wnt signaling. Embo J. 2002;21:2703-2714. 